\title{
On the absence of trapped modes in locally perturbed open waveguides
}

\author{
Christophe Hazard \\ Laboratoire de Propagation d'Ondes : Etude Mathématique et Simulation, \\ Ecole Nationale Supérieure de Techniques Avancées, \\ 828 boulevard des Maréchaux, 91120 Palaiseau, France \\ christophe.hazard@ensta-paristech.fr
}

March 31, 2014

\begin{abstract}
This paper presents a new approach for proving that the presence of a bounded defect in a uniform open waveguide cannot produce trapped modes, contrary to the case of a closed waveguide. The originality of the proof lies in the fact that it relies on a modal decomposition. It shows in particular that the absence of trapped modes results from a strong connection between the various modal components of the field. The case of the three-dimensional scalar wave equation is considered.
\end{abstract}

Keywords: Open waveguide, Helmholtz equation, generalized Fourier transform.

\section{Introduction}

\subsection{About trapped modes}

In a non-dissipative propagative medium filling an unbounded part of the space, trapped modes, also referred to as resonance modes or bound states, designate localized free oscillations of the medium which do not radiate towards infinity. More precisely, these are time-harmonic solutions of the propagation equations which have a finite energy that remains confined in a bounded region despite the unboundedness of the propagative medium. In this paper, we wonder whether such trapped modes can occur in locally perturbed open waveguides.

A uniform cylindrical waveguide is a device whose physical features are invariant in one space direction, called here longitudinal, and which is able to propagate waves over long distances in this direction. Such a waveguide is called closed if it is bounded in the transverse directions by an impenetrable wall, and open if its cross-section can be considered as unbounded, as for instance optical fibers, immersed pipes, or other guiding devices embedded into a propagative matrix. Actually the term 'waveguide' is justified by the existence of guided waves, that is, time-harmonic waves that propagate without attenuation in the longitudinal direction and remain confined in the other transverse directions. For a closed waveguide, such a confinement is simply due to the boundedness of the cross-section. But for an open waveguide, this confinement results from a particular layout of the various materials which compose the waveguide. Numerous papers are devoted to the existence and the properties of guided modes for acoustic, elastic or electromagnetic open waveguides (see, e.g., [5, 7] and references cited therein).

Here we are rather interested in trapped modes, which remain confined in both transverse and longitudinal directions. Trapped modes generally do not occur in cylindrical uniform waveguides, simply because there is nowhere to confine energy. More exactly, because of the longitudinal invariance, if a trapped mode existed, any translation of this mode in the longitudinal direction would remain trapped, which would yield an infinite-dimensional space of trapped modes. This may occur in very exceptional situations which are not considered here (for instance at the interface between a dielectric and a metamaterial [12]). In fact, a natural way to allow the energy confinement is to break the longitudinal invariance 
by introducing a local perturbation, that is, a bounded defect, in a uniform cylindrical waveguide. For closed waveguides, it is now well understood that such local perturbations may produce trapped modes (see [16] for a review). In a similar vein, there is an extensive literature about the occurrence of trapped modes in curved closed waveguides (see, e.g., [11,9]). Those who are familiar with closed waveguides could be surprised to discover that the case of open waveguides differs singularly. Actually the pioneer work of Weder [21, 22] shows that no trapped mode can occur in locally perturbed stratified media. This result was then extended to more general situations in [10], using an abstract argument which became very popular during the last decades in the mathematical physics community: the Mourre's commutator theory.

The purpose of the present paper is to explore an alternative proof for the absence of trapped modes in a locally perturbed open waveguide. The final result is not new but the proof is. Its originality lies in the tools we use, which are natural and usual tools for physicists, contrary to the abstract technique proposed in [10]. Actually the proof proposed here relies on a modal decomposition which consists in representing any wave as a superposition of modes. It shows in particular that the absence of trapped modes results from a strong connection between the various modal components.

\subsection{The mathematical model}

We consider acoustic waveguides filling the whole space $\mathbb{R}^{3}$ equipped with an orthonormal coordinate system $\left(x, y_{1}, y_{2}\right)$, where $x \in \mathbb{R}$ represents the longitudinal direction, that is, the privileged direction of propagation, and $y:=\left(y_{1}, y_{2}\right) \in \mathbb{R}^{2}$, the transverse directions. We are interested in the solutions $u=u(x, y)$ to the time-harmonic acoustic wave equation

$$
-\Delta u-k^{2} u=0 \quad \text { in } \mathbb{R}^{3}
$$

where $k=k(x, y)$ is a real wavenumber function assumed such that

$$
0<k_{\mathrm{m}} \leq k(x, y) \leq k_{\mathrm{M}}<+\infty \quad \forall(x, y) \in \mathbb{R}^{3},
$$

for two positive constants $k_{\mathrm{m}}$ and $k_{\mathrm{M}}$.

In the particular case of a uniform cylindrical waveguide, this wavenumber function, denoted by $k_{\text {uni }}$, depends only on the transverse variable and we suppose moreover that $k_{\text {uni }}$ is constant outside a bounded region, i.e.,

$$
k_{\text {uni }}=k_{\text {uni }}(y) \quad \text { with } \quad k_{\text {uni }}(y)=k_{\infty}>0 \quad \text { if }|y|:=\left(y_{1}^{2}+y_{2}^{2}\right)^{1 / 2}>d
$$

for some $d>0$. The inhomogeneous region represents the core of the waveguide. The above assumption means that the surrounding cladding is homogeneous.

We are interested in the case where $k$ is a localized perturbation of such a function $k_{\text {uni }}$ in the sense that

$$
k-k_{\text {uni }} \text { is compactly supported. }
$$

Such a function $k$ describes a uniform waveguide perturbed locally by the presence of a bounded penetrable defect (see Figure 1).

Trapped modes are defined as non-zero finite-energy solutions to (1), which amounts here to assuming that $u \in L^{2}\left(\mathbb{R}^{3}\right)$, where $L^{2}$ denotes the usual space of square integrable functions. Our aim is to prove the following theorem, which asserts the absence of such modes.

Theorem 1. Let $k$ and $k_{\text {uni }}$ satisfy assumptions (2), (3) and (4). Then the only solution $u \in L^{2}\left(\mathbb{R}^{3}\right)$ to the Helmholtz equation (1) (in the distribution sense) is $u \equiv 0$.

\subsection{Guiding ideas of the proof}

The scheme of the proof follows and extends the approach proposed in [3] for studying the scattering by a defect in a two-dimensional step-index waveguide. The keystone of this approach lies in the use of a modal decomposition of the acoustic field in the transverse directions. Such a decomposition is often used for closed waveguides. In this case, the acoustic field appears as the sum of a finite superposition 


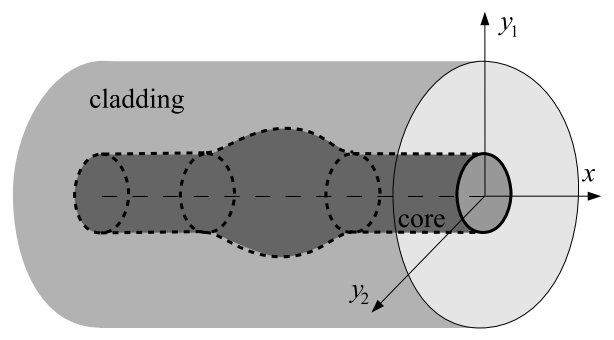

Figure 1: A locally perturbed uniform waveguide

(possibly empty) of guided modes and an infinite superposition of evanescent modes (see, e.g., [20]). On the other hand, for open waveguides, such a modal decomposition still exists but it involves a continuum. The acoustic field appears as the sum of a finite superposition of guided modes, which are evanescent in the transverse directions, and a continuous superposition of radiation modes, which are so called because they are oscillating in the transverse directions. This continuum actually consists of two parts according to the longitudinal behavior of the modes, which can be either oscillating or evanescent. Those which are oscillating can be classified within a category of propagative modes which also contains the finite set of guided modes, since both kinds propagate without attenuation in the longitudinal direction.

When looking at the modal decomposition of a trapped mode, a simple energy argument tells us that outside any transverse section which contains the defect of our waveguide, all the modal components associated with these propagative modes must vanish. In fact, if some of these components were not zero, the energy of the trapped mode would be infinite, since propagative modes carry energy towards infinity. Then a more subtle argument enters the scene: because of the boundedness of the defect, the modal components extend to an analytic function of the modal parameter (which is related to the longitudinal wavenumber). It seems difficult to propose a physical interpretation of this analyticity property which provides us the key to the riddle. Indeed, as we already know that the modal components vanish on a continuous interval, analyticity implies that they must vanish everywhere: the modal components associated with evanescent modes are also zero. We conclude that the only finite-energy solution to the propagation equation is zero: trapped modes do not exist.

It is worth noticing that this approach cannot be used for a closed waveguide. In this case, the energy argument shows as above that the modal components associated with propagative modes must vanish. But these components compose a finite set, so the analyticity argument cannot apply. Actually, nothing can be said about the components associated with evanescent modes. This is precisely why trapped modes can occur in closed waveguides. To a certain extent, in an open waveguide, the above analyticity property means that the propagative and evanescent components are connected in a subtle but strong way, whereas they are independent in a closed waveguide.

This very powerful argument of analyticity seems to have been used for the first time by Littman [17] for proving the absence of eigenvalues in the continuous spectrum of the Laplacian outside a deformed cylinder. Shortly after, it was used independently by Weder [21] for proving the absence of trapped modes for a perturbed stratified medium. The approach proposed by Weder is based on the usual Fourier transform in the direction of the layers. In [3], we adopted a similar but orthogonal point of view. Indeed the above mentioned modal decomposition amounts to using a generalized Fourier transform in the transverse direction, which simplifies considerably the proof and allows to deal with more complex situations such as the junction of two open waveguides, as shown in [6]. In the latter papers, we investigated the time-harmonic scattering problem, not only the question of absence of trapped modes (which appears as a by-product of the solvability of the scattering problem). But for a technical reason which is related to the possibility of extending the generalized Fourier transform to non- $L^{2}$ fields [14], we restricted ourselves to very simple two-dimensional step-index open waveguides. In the present paper, the fact that we are interested only in trapped modes allows us to deal with more general waveguides, for which the question of the solvability of the scattering problem remains open.

The paper is organized as follows. In section 2, we introduce the main ingredients which provide us the modal decomposition of the acoustic field. The proof of Theorem 1 is the subject of section 3 . We finally present in section 4 some possible extensions of Theorem 1. 
In the whole paper, $\sqrt{\cdot}$ denotes the following determination of the complex square root:

$$
\sqrt{\zeta}:=|\zeta|^{1 / 2} \mathrm{e}^{\mathrm{i}(\arg \zeta) / 2} \quad \text { for } \zeta \in \mathbb{C} \text { with }-3 \pi / 2<\arg \zeta \leq \pi / 2 .
$$

\section{Modal analysis of a uniform waveguide}

\subsection{Spectrum of the transverse Helmholtz operator}

As mentioned in the introduction, our approach is mainly based on a modal analysis of a uniform open waveguide which allows us to represent a time-harmonic wave as the sum of a finite superposition of guided modes and a continuous superposition of radiation modes. These modes are simply obtained by the method of separation of variables applied to the Helmholtz equation (1) with $k$ replaced by $k_{\text {uni }}$. Setting $u(x, y)=\varphi(y) \exp (\gamma x)$ for some complex number $\gamma$, we are led to find $\lambda:=\gamma^{2} \in \mathbb{C}$ and a non-zero function $\varphi$ defined in $\mathbb{R}^{2}$ such that

$$
-\Delta_{y} \varphi-k_{\text {uni }}^{2} \varphi=\lambda \varphi \text { in } \mathbb{R}^{2},
$$

where $\Delta_{y}$ denotes the two-dimensional Laplace operator in the $y$-plane. In other words, we search for the eigenelements of the unbounded selfadjoint operator $A$ defined in $L^{2}\left(\mathbb{R}^{2}\right)$ by

$$
A \varphi:=-\Delta_{y} \varphi-k_{\text {uni }}^{2} \varphi \quad \forall \varphi \in \mathrm{D}(A):=H^{2}\left(\mathbb{R}^{2}\right),
$$

which represents the transverse part of the Helmholtz operator. Here, $H^{2}$ denotes the usual Sobolev space of order 2. It is well-known (see, e.g., [4]) that the spectrum $\Lambda$ of $A$ is composed of two parts: a continuous spectrum $\Lambda_{\mathrm{c}}:=\left[-k_{\infty}^{2},+\infty\right)$ and a finite point spectrum $\Lambda_{\mathrm{p}}$ which is non-empty if and only if

$$
k_{*}:=\sup _{y \in \mathbb{R}^{2}} k_{\text {uni }}(y)>k_{\infty},
$$

which means that somewhere in the core, the wave speed is smaller than in the cladding. In this case, we have $\Lambda_{\mathrm{p}} \subset\left(-k_{*}^{2},-k_{\infty}^{2}\right)$ and every eigenvalue $\lambda \in \Lambda_{\mathrm{p}}$ has a finite multiplicity.

Remark 2. Inequality (7) is a necessary and sufficient condition to ensure the existence of guided modes, which legitimate the use of the word 'waveguide' throughout this paper. However this assumption is not crucial in our analysis. Our results hold true for non-guiding devices.

What about eigenfunctions? On the one hand, if $\lambda \in \Lambda_{\mathrm{p}}$, the associated eigenfunctions $\varphi$ belong by definition to $H^{2}\left(\mathbb{R}^{2}\right)$ : they actually decay exponentially as $|y| \rightarrow \infty$. The corresponding modes $\varphi(y) \exp ( \pm \sqrt{\lambda} x)$ are called guided modes for they propagate without attenuation in the longitudinal direction $x$ (since $\sqrt{\lambda}$ is purely imaginary) whereas in the transverse directions, they remain confined near the core. On the other hand, if $\lambda \in \Lambda_{\mathrm{c}}$, the associated solutions $\varphi$ are usually called generalized eigenfunctions for they no longer belong to $L^{2}\left(\mathbb{R}^{2}\right)$ (otherwise, $\lambda$ would be an eigenvalue). Actually, these functions are oscillating as $|y| \rightarrow \infty$. Because of this transverse behavior, the corresponding modes $\varphi(y) \exp ( \pm \sqrt{\lambda} x)$ are referred to as radiation modes. They are propagative in the longitudinal direction if $\lambda \in\left(-k_{\infty}^{2}, 0\right)$ but exponentially increasing or decreasing if $\lambda>0$.

It seems natural to wonder if the knowledge of the above mentioned eigenfunctions and generalized eigenfunctions allows us to diagonalize our operator $A$. More precisely, can one build a generalized spectral basis associated with $A$, that is, a complete family of eigenfunctions and generalized eigenfunctions in which $A$ becomes diagonal. Although the signification of both words 'complete' and 'diagonal' is somewhat imprecise at this stage, one can assert that such a construction is always possible for any selfadjoint operator in a Hilbert space. This result can be seen as an advanced version of the spectral theorem [2]. However the practical construction of a generalized spectral basis highly depends on the operator and its rigorous justification is in general a difficult task. In order to understand such a construction in our situation, we begin with the case of a homogeneous medium which easily derives from the usual Fourier transform. 


\subsection{Diagonalization: the homogeneous case}

Consider the operator $A_{\infty}$ defined as in (6) with $k_{\text {uni }}$ replaced by $k_{\infty}$, that is, $A_{\infty}:=-\Delta_{y}-k_{\infty}^{2}$. Of course its spectrum is purely continuous and equal to $\Lambda_{\mathrm{c}}=\left[-k_{\infty}^{2},+\infty\right)$. Let $\mathcal{F}$ denote the usual Fourier transform in the $y$-plane defined by

$$
\mathcal{F} \varphi(\xi):=\frac{1}{2 \pi} \int_{\mathbb{R}^{2}} \varphi(y) \mathrm{e}^{-\mathrm{i} \xi \cdot y} \mathrm{~d} y \quad \forall \xi \in \mathbb{R}^{2},
$$

which is unitary from $L^{2}\left(\mathbb{R}_{y}^{2}\right)$ to $L^{2}\left(\mathbb{R}_{\xi}^{2}\right)$. We clearly have

$$
\left(\mathcal{F} A_{\infty} \varphi\right)(\xi)=\left(|\xi|^{2}-k_{\infty}^{2}\right) \mathcal{F} \varphi(\xi) \quad \forall \varphi \in \mathrm{D}\left(A_{\infty}\right):=H^{2}\left(\mathbb{R}^{2}\right) .
$$

It is then natural to introduce the change of variables

$$
\mathbb{R}^{2} \ni \xi \mapsto(\lambda, \kappa):=\left(|\xi|^{2}-k_{\infty}^{2}, \frac{\xi}{|\xi|}\right) \in \Lambda_{\mathrm{c}} \times S^{1},
$$

where $S^{1}$ denotes the unit circle in $\mathbb{R}^{2}$. Noticing that

$$
\int_{\mathbb{R}^{2}}|\psi(\xi)|^{2} \mathrm{~d} \xi=\frac{1}{2} \int_{-k_{\infty}^{2}}^{+\infty} \int_{S^{1}}\left|\psi\left(\kappa \sqrt{k_{\infty}^{2}+\lambda}\right)\right|^{2} \mathrm{~d} \lambda \mathrm{d} s_{\kappa} \quad \forall \psi \in L^{2}\left(\mathbb{R}_{\xi}^{2}\right),
$$

we infer that the transformation $\mathcal{C}$ defined by

$$
\psi(\xi) \mapsto \mathcal{C} \psi(\lambda, \kappa):=\frac{1}{\sqrt{2}} \psi\left(\kappa \sqrt{k_{\infty}^{2}+\lambda}\right)
$$

is unitary from $L^{2}\left(\mathbb{R}_{\xi}^{2}\right)$ to $L^{2}\left(\Lambda_{\mathrm{c}} \times S^{1}\right)$. Hence, $\mathcal{U}_{\infty}:=\mathcal{C F}$ is unitary from $L^{2}\left(\mathbb{R}_{y}^{2}\right)$ to $L^{2}\left(\Lambda_{\mathrm{c}} \times S^{1}\right)$. Moreover, we deduce from (9) that

$$
A_{\infty} \varphi=\mathcal{U}_{\infty}^{-1} \lambda \mathcal{U}_{\infty} \varphi \quad \forall \varphi \in \mathrm{D}\left(A_{\infty}\right),
$$

where $\lambda$ stands here for the operator of multiplication by $\lambda$ in $L^{2}\left(\Lambda_{\mathrm{c}} \times S^{1}\right)$. The latter formula means that $\mathcal{U}_{\infty}$ diagonalizes $A_{\infty}$ in the spectral space $L^{2}\left(\Lambda_{\mathrm{c}} \times S^{1}\right)$. From (8) and (10), this unitary operator can be rewritten in the convenient form

$$
\begin{aligned}
\mathcal{U}_{\infty} \varphi(\lambda, \kappa) & =\int_{\mathbb{R}^{2}} \varphi(y) \overline{\Phi_{\lambda, \kappa}^{\infty}(y)} \mathrm{d} y \quad \text { where } \\
\Phi_{\lambda, \kappa}^{\infty}(y) & :=\frac{1}{\sqrt{8} \pi} \exp \left(\mathrm{i} \sqrt{k_{\infty}^{2}+\lambda} \kappa \cdot y\right) \quad \forall(\lambda, \kappa) \in \Lambda_{\mathrm{c}} \times S^{1} .
\end{aligned}
$$

Function $\Phi_{\lambda, \kappa}^{\infty}$ represents a plane wave of direction $\kappa$. It is a generalized eigenfunction of $A_{\infty}$ associated with the spectral value $\lambda$ in the sense that it satisfies

$$
\left(-\Delta_{y}-k_{\infty}^{2}\right) \Phi_{\lambda, \kappa}^{\infty}=\lambda \Phi_{\lambda, \kappa}^{\infty} \quad \text { in } \mathbb{R}^{2},
$$

but it does not belong to $L^{2}\left(\mathbb{R}^{2}\right)$. And $\mathcal{U}_{\infty}$ appears as the operator of 'decomposition' on the family $\left\{\Phi_{\lambda, \kappa}^{\infty} ;(\lambda, \kappa) \in \Lambda_{\mathrm{c}} \times S^{1}\right\}$ which can be seen as a generalized spectral basis for $A_{\infty}$.

\subsection{Diagonalization: the perturbed case}

We come back now to the transverse operator $A$ defined in (6). The formal construction of a generalized spectral basis for $A$ simply consists in a perturbation of our generalized spectral basis for $A_{\infty}$, where each $\Phi_{\lambda, \kappa}^{\infty}$ defined in (12) is interpreted as an incident plane wave which is scattered by the core. Hence we search for generalized eigenfunctions of $A$ in the form

$$
\Phi_{\lambda, \kappa}:=\Phi_{\lambda, \kappa}^{\infty}+\Phi_{\lambda, \kappa}^{\mathrm{sc}} \quad \text { for }(\lambda, \kappa) \in \Lambda_{\mathrm{c}} \times S^{1},
$$


where $\Phi_{\lambda, \kappa}^{\mathrm{sc}}$ stands for the associated scattered wave, which is the solution to

$$
\begin{aligned}
& -\Delta_{y} \Phi_{\lambda, \kappa}^{\mathrm{sc}}-\left(k_{\mathrm{uni}}^{2}+\lambda\right) \Phi_{\lambda, \kappa}^{\mathrm{sc}}=\left(k_{\mathrm{uni}}^{2}-k_{\infty}^{2}\right) \Phi_{\lambda, \kappa}^{\infty} \quad \text { in } \mathbb{R}^{2}, \\
& \frac{\partial \Phi_{\lambda, \kappa}^{\mathrm{sc}}}{\partial|y|}-\mathrm{i} \sqrt{k_{\infty}^{2}+\lambda} \Phi_{\lambda, \kappa}^{\mathrm{sc}}=O\left(|y|^{-3 / 2}\right) \quad \text { as }|y| \rightarrow \infty .
\end{aligned}
$$

Recall that the outgoing radiation condition (16) ensures the uniqueness of the solution $\Phi_{\lambda, \kappa}^{\mathrm{sc}}$ to (15) (see, e.g., [8]). Note that instead of this condition, one could choose the incoming condition obtained by replacing $-\mathrm{i}$ by $+\mathrm{i}$ : the results given below hold true. Each $\Phi_{\lambda, \kappa}$ is a generalized eigenfunction of $A$ associated with $\lambda$ for it satisfies by construction

$$
\left(-\Delta_{y}-k_{\text {uni }}^{2}\right) \Phi_{\lambda, \kappa}=\lambda \Phi_{\lambda, \kappa} \quad \text { in } \mathbb{R}^{2},
$$

but it does not belong to $L^{2}\left(\mathbb{R}^{2}\right)$. We actually have $\Phi_{\lambda, \kappa} \in H_{\text {loc }}^{2}\left(\mathbb{R}^{2}\right)$, i.e., $\left.\Phi_{\lambda, \kappa}\right|_{\mathcal{O}} \in H^{2}(\mathcal{O})$ for all bounded $\mathcal{O} \subset \mathbb{R}^{2}$

When the point spectrum $\Lambda_{\mathrm{p}}$ is not empty (see Remark 2), this continuous family of generalized eigenfunctions must be completed by a finite family of eigenfunctions which simply consists of the union of orthonormal bases of the various eigenspaces. For the sake of conciseness, we use the same notation $\Phi_{\lambda, \kappa}$ for both families: if $\lambda$ is an eigenvalue of multiplicity $m_{\lambda}$, we denote by $\Phi_{\lambda, \kappa} \in H^{2}\left(\mathbb{R}^{2}\right)$ for $\kappa=1$ to $m_{\lambda}$, an orthonormal basis of the associated eigenspace. This leads us to consider the completed family defined by

$$
\left\{\Phi_{\lambda, \kappa} ; \lambda \in \Lambda, \kappa \in K_{\lambda}\right\} \quad \text { where } \quad K_{\lambda}:= \begin{cases}\left\{1, \ldots, m_{\lambda}\right\} \subset \mathbb{N} & \text { if } \lambda \in \Lambda_{\mathrm{p}}, \\ S^{1} & \text { if } \lambda \in \Lambda_{\mathrm{c}} .\end{cases}
$$

The fact that this family constitutes a generalized spectral basis can be formulated by considering the analogue of operator $\mathcal{U}_{\infty}$ defined in (11), that is, the operator of 'decomposition' on this family, given by

$$
\mathcal{U} \varphi(\lambda, \kappa):=\int_{\mathbb{R}^{2}} \varphi(y) \overline{\Phi_{\lambda, \kappa}(y)} \mathrm{d} y \quad \forall \lambda \in \Lambda, \forall \kappa \in K_{\lambda} .
$$

If $\lambda \in \Lambda_{\mathrm{p}}$, the above integral is simply the $L^{2}$ inner product of $\varphi$ by the eigenfunction $\Phi_{\lambda, \kappa} \in L^{2}\left(\mathbb{R}^{2}\right)$. On the other hand, if $\lambda \in \Lambda_{\mathrm{c}}$, this definition does not make sense for all $\varphi \in L^{2}\left(\mathbb{R}^{2}\right)$ since $\Phi_{\lambda, \kappa}$ no longer belongs to $L^{2}\left(\mathbb{R}^{2}\right)$. It applies for instance to compactly supported $\varphi$. As for the usual Fourier transform, a density argument shows that this definition extends to all functions $\varphi \in L^{2}\left(\mathbb{R}^{2}\right)$. This result is part of the following proposition, which collects the main properties of $\mathcal{U}$. Its proof dates back to the early seventies [18] and can be generalized for numerous scattering problems [13].

Proposition 3. (i) The operator $\mathcal{U}$ defined by (17) for compactly supported $\varphi \in L^{2}\left(\mathbb{R}^{2}\right)$ extends (by density) to a unitary transformation from $L^{2}\left(\mathbb{R}^{2}\right)$ to the spectral space

$$
\widehat{\mathcal{H}}:=\widehat{\mathcal{H}}_{\mathrm{p}} \oplus \widehat{\mathcal{H}}_{\mathrm{c}} \quad \text { where } \quad \widehat{\mathcal{H}}_{\mathrm{p}}:=\bigoplus_{\lambda \in \Lambda_{\mathrm{p}}} \mathbb{C}^{m_{\lambda}} \quad \text { and } \quad \widehat{\mathcal{H}}_{\mathrm{c}}:=L^{2}\left(\Lambda_{\mathrm{c}} \times S^{1}\right) \text {. }
$$

(ii) The inverse transform $\mathcal{U}^{-1}$ appears as the operator of 'recomposition' on the family $\left\{\Phi_{\lambda, \kappa} ; \lambda \in\right.$ $\left.\Lambda, \kappa \in K_{\lambda}\right\}:$ for all $\widehat{\varphi} \in \widehat{\mathcal{H}}$,

$$
\mathcal{U}^{-1} \widehat{\varphi}=\sum_{\lambda \in \Lambda_{\mathrm{p}}} \sum_{\kappa=1}^{m_{\lambda}} \widehat{\varphi}(\lambda, \kappa) \Phi_{\lambda, \kappa}+\int_{\Lambda_{\mathrm{c}} \times S^{1}} \widehat{\varphi}(\lambda, \kappa) \Phi_{\lambda, \kappa} \mathrm{d} \lambda \mathrm{d} s_{\kappa} .
$$

(iii) $\mathcal{U}$ diagonalizes $A$ in the sense that

$$
A \varphi=\mathcal{U}^{-1} \lambda \mathcal{U} \varphi \quad \forall \varphi \in \mathrm{D}(A),
$$

where $\lambda$ denotes here the operator of multiplication by $\lambda$ in $\widehat{\mathcal{H}}$.

Operator $\mathcal{U}$ is often called a generalized Fourier transform associated with $A$. Item (i) shows that the perturbation process described above yields a generalized spectral basis of $L^{2}\left(\mathbb{R}^{2}\right)$. Item (ii), which is not used in the present paper, extends the well-known expression of the usual inverse Fourier transform. And item (iii) provides the key for a functional calculus of $A$ since any function of $A$ can be simply defined by $f(A)=\mathcal{U}^{-1} f(\lambda) \mathcal{U}$. 


\subsection{Analyticity of the generalized eigenfunctions}

In addition to the latter proposition, we shall need a basic property of the generalized eigenfunctions expressed as follows.

Proposition 4. Let $\Omega$ be a bounded open subset of $\mathbb{R}^{2}$ and $\kappa \in S^{1}$. The function $\left(-k_{\infty}^{2},+\infty\right) \ni \lambda \mapsto$ $\left.\Phi_{\lambda, \kappa}\right|_{\Omega} \in L^{2}(\Omega)$ extends to a meromorphic function of $\lambda$ in the complex half plane $\operatorname{Re} \lambda>-k_{\infty}^{2}$. And the same holds for the function $\left(-k_{\infty}^{2},+\infty\right) \ni \lambda \mapsto \overline{\Phi_{\lambda, \kappa}} \in L^{2}(\Omega)$.

Proof. For $\lambda \in\left(-k_{\infty}^{2},+\infty\right)$, let $G_{\lambda}$ denote the outgoing Green's function associated with equation (13), which is given by

$$
G_{\lambda}(y):=\frac{\mathrm{i}}{4} H_{0}^{(1)}\left(\sqrt{k_{\infty}^{2}+\lambda}|y|\right)
$$

where $H_{0}^{(1)}$ is the Hankel function of the first kind and order 0. It is well-known (see, e.g., [8]) that the definition (14)-(16) of the generalized eigenfunctions is equivalent to the Lippmann-Schwinger equation

$$
\Phi_{\lambda, \kappa}(y)-\int_{\mathbb{R}^{2}} G_{\lambda}\left(y-y^{\prime}\right)\left(k_{\text {uni }}^{2}\left(y^{\prime}\right)-k_{\infty}^{2}\right) \Phi_{\lambda, \kappa}\left(y^{\prime}\right) \mathrm{d} y^{\prime}=\Phi_{\lambda, \kappa}^{\infty}(y) \quad \forall y \in \mathbb{R}^{2} .
$$

In this equation, the convolution term represents the scattered wave. The associated operator $V_{\lambda}$ defined by

$$
V_{\lambda} \varphi(y):=\int_{\mathbb{R}^{2}} G_{\lambda}\left(y-y^{\prime}\right)\left(k_{\text {uni }}^{2}\left(y^{\prime}\right)-k_{\infty}^{2}\right) \varphi\left(y^{\prime}\right) \mathrm{d} y^{\prime} \quad \forall y \in \mathbb{R}^{2},
$$

is continuous from $L^{2}\left(\mathbb{R}^{2}\right)$ to $H^{2}(\Omega)$ for every bounded domain $\Omega \subset \mathbb{R}^{2}$. Hence, if $\Omega$ contains the support of $k_{\mathrm{uni}}^{2}-k_{\infty}^{2}, V_{\lambda}$ appears as a compact operator in $L^{2}(\Omega)$.

Moreover, as $H_{0}^{(1)}$ is analytic in $\mathbb{C} \backslash \mathbb{R}^{-}$, the above definition of $V_{\lambda}$ extends to complex values of $\lambda$. Using for instance the definition (5) of the complex square root, one can readily show that $\left\{V_{\lambda} ; \operatorname{Re} \lambda>-k_{\infty}^{2}\right\}$ defines an analytic family of compact operators in $L^{2}(\Omega)$ and that $\left\{\Phi_{\lambda, \kappa}^{\infty} ; \operatorname{Re} \lambda>-k_{\infty}^{2}\right\}$ is an analytic family of vectors of $L^{2}(\Omega)$. The analytic Fredholm theory $[19,8]$ then tells us that either $\mathrm{I}-V_{\lambda}$ is not invertible for any $\lambda$ or its inverse $\left(\mathrm{I}-V_{\lambda}\right)^{-1}$ is meromorphic in $\operatorname{Re} \lambda>-k_{\infty}^{2}$. The former situation must be excluded. Indeed, if $\operatorname{Im} \lambda>0$, then $G_{\lambda}(y)$ is exponentially decreasing as $|y| \rightarrow \infty$, thus it is easily seen that equation (19) is equivalent to solve

$$
(A-\lambda \mathrm{I}) \Phi_{\lambda, \kappa}^{\mathrm{sc}}=\left(k_{\mathrm{uni}}^{2}-k_{\infty}^{2}\right) \Phi_{\lambda, \kappa}^{\infty} \quad \text { in } L^{2}\left(\mathbb{R}^{2}\right)
$$

which is a well-posed equation since $\lambda \notin \Lambda$. As a consequence, the solution $\Phi_{\lambda, \kappa}$ to (19) is meromorphic and its poles, which are related to the so-called 'leaky modes' (see, e.g., [20]), are necessarily contained in $\operatorname{Im} \lambda<0$.

For the function $\lambda \mapsto \overline{\Phi_{\lambda, \kappa}(y)}$, we can use the same arguments starting with the conjugate of the Lippmann-Schwinger equation (19). In this case, the poles are contained in $\operatorname{Im} \lambda>0$.

\section{Proof of Theorem 1}

The hardest part of the job is now done! Thanks to the generalized Fourier transform $\mathcal{U}$, the proof of Theorem 1 becomes easy. For the sake of clarity, it is divided into three steps.

\subsection{First step: getting rid of the defect}

The starting point of the proof simply consists in noticing that if $u \in L^{2}\left(\mathbb{R}^{3}\right)$ is a solution to the homogeneous Helmholtz equation (1), then

$$
-\Delta u-k_{\text {uni }}^{2} u=f(u) \text { in } \mathbb{R}^{3},
$$

where $f(u):=\left(k^{2}-k_{\text {uni }}^{2}\right) u \in L^{2}\left(\mathbb{R}^{3}\right)$ is compactly supported. The aim of the next sections is to prove the following property related to the uniform waveguide. 
Theorem 5. Let $f \in L^{2}\left(\mathbb{R}^{3}\right)$ be compactly supported. If $u \in L^{2}\left(\mathbb{R}^{3}\right)$ satisfies

$$
-\Delta u-k_{\text {uni }}^{2} u=f \quad \text { in } \mathbb{R}^{3},
$$

then $u=0$ outside the support of $f$.

Theorem 1 is actually a corollary of the latter: it suffices to apply the unique continuation principle [15] to equation (1), which tells us that $u$ also vanishes inside the support of $f$.

So, by reducing the proof of Theorem 1 to that of Theorem 5 , we got rid of the defect. It remains to prove Theorem 20. In the sequel, $f$ denotes a function of $L^{2}\left(\mathbb{R}^{3}\right)$ compactly supported and $u \in L^{2}\left(\mathbb{R}^{3}\right)$, a solution to (20). From the definition (6) of $A$, we can rewrite this equation in the following abstract form:

$$
-\frac{\partial^{2} u}{\partial x^{2}}+A u=f \quad \text { in } \mathbb{R} .
$$

\subsection{Second step: searching for finite-energy solutions}

As both functions $u$ and $f$ belong to $L^{2}\left(\mathbb{R}^{3}\right)$, we know that $u(x, \cdot)$ and $f(x, \cdot)$ belong to $L^{2}\left(\mathbb{R}^{2}\right)$ for almost every $x \in \mathbb{R}$ so that we can define their partial generalized Fourier transforms by

$$
\widehat{u}_{\lambda, \kappa}(x):=(\mathcal{U} u(x, \cdot))(\lambda, \kappa) \text { and } \widehat{f}_{\lambda, \kappa}(x):=(\mathcal{U} f(x, \cdot))(\lambda, \kappa) .
$$

Thanks to the notion of tensor products of Hilbert spaces (see, e.g., [1]) and the fact that $\mathcal{U}$ is unitary from $L^{2}\left(\mathbb{R}^{2}\right)$ to $\widehat{\mathcal{H}}$ (Proposition 3), we know that these functions, considered as functions of $(x, \lambda, \kappa)$, belong to $L^{2}(\mathbb{R}) \otimes \widehat{\mathcal{H}}$. This implies in particular that

$$
\widehat{u}_{\lambda, \kappa} \in L^{2}(\mathbb{R}) \text { for a.e. } \lambda \in \Lambda \text { and } \kappa \in K_{\lambda} \text {. }
$$

Note that the expression 'for a.e. $\lambda \in \Lambda^{\prime}$ ' (for almost every) is relative to the spectral measure: it means 'for all $\lambda \in \Lambda_{\mathrm{p}} \cup\left(\Lambda_{\mathrm{c}} \backslash \Lambda_{0}\right)$ ' where $\Lambda_{0} \subset \Lambda_{\mathrm{c}}$ has Lebesgue measure zero.

Applying $\mathcal{U}$ to equation (21) and using (18) then yields

$$
-\frac{\partial^{2} \widehat{u}_{\lambda, \kappa}}{\partial x^{2}}+\lambda \widehat{u}_{\lambda, \kappa}=\widehat{f}_{\lambda, \kappa} \quad \text { in } \mathbb{R} \text {, for a.e. } \lambda \in \Lambda \text { and } \kappa \in K_{\lambda} .
$$

The solutions to this differential equation can be decomposed as

$$
\widehat{u}_{\lambda, \kappa}=\widehat{u}_{\lambda, \kappa}^{\mathrm{gen}}+\widehat{u}_{\lambda, \kappa}^{\mathrm{par}}
$$

where $\widehat{u}_{\lambda, \kappa}^{\text {gen }}$ denotes the general solution to the homogeneous equation (i.e., when $\widehat{f}_{\lambda, \kappa}=0$ ) which is given by

$$
\widehat{u}_{\lambda, \kappa}^{\text {gen }}(x):=\widehat{\alpha}_{\lambda, \kappa}^{-} \mathrm{e}^{-\sqrt{\lambda} x}+\widehat{\alpha}_{\lambda, \kappa}^{+} \mathrm{e}^{+\sqrt{\lambda} x} \quad \text { with } \widehat{\alpha}_{\lambda, \kappa}^{ \pm} \in \mathbb{C},
$$

and $\widehat{u}_{\lambda, \kappa}^{\mathrm{par}}$ is a particular solution to (23) defined by

$$
\widehat{u}_{\lambda, \kappa}^{\mathrm{par}}(x):=\int_{\mathbb{R}} \gamma_{\lambda}\left(x-x^{\prime}\right) \widehat{f}_{\lambda, \kappa}\left(x^{\prime}\right) \mathrm{d} x^{\prime} \quad \text { where } \quad \gamma_{\lambda}(x):=\frac{\mathrm{e}^{-\sqrt{\lambda}|x|}}{2 \sqrt{\lambda}}
$$

is a Green's function of the operator $-\partial^{2} / \partial x^{2}+\lambda$. Let $\ell>0$ such that the support of $f$ is contained in the strip $|x| \leq \ell$. We deduce from the above expression that

$$
\widehat{u}_{\lambda, \kappa}^{\mathrm{par}}(x)=\widehat{\beta}_{\lambda, \kappa}^{ \pm} \mathrm{e}^{-\sqrt{\lambda}|x|} \text { if } \pm x>\ell, \quad \text { where } \quad \widehat{\beta}_{\lambda, \kappa}^{ \pm}:=\int_{-\ell}^{+\ell} \frac{\mathrm{e}^{ \pm \sqrt{\lambda} x^{\prime}}}{2 \sqrt{\lambda}} \widehat{f}_{\lambda, \kappa}\left(x^{\prime}\right) \mathrm{d} x^{\prime} .
$$

As a consequence

$$
\widehat{u}_{\lambda, \kappa}(x)= \begin{cases}\widehat{\alpha}_{\lambda, \kappa}^{-} \mathrm{e}^{-\sqrt{\lambda} x}+\left(\widehat{\alpha}_{\lambda, \kappa}^{+}+\widehat{\beta}_{\lambda, \kappa}^{-}\right) \mathrm{e}^{+\sqrt{\lambda} x} & \text { if } x<-\ell, \\ \left(\widehat{\alpha}_{\lambda, \kappa}^{-}+\widehat{\beta}_{\lambda, \kappa}^{+}\right) \mathrm{e}^{-\sqrt{\lambda} x}+\widehat{\alpha}_{\lambda, \kappa}^{+} \mathrm{e}^{+\sqrt{\lambda} x} & \text { if } x>+\ell\end{cases}
$$


First consider the case of propagative modes, that is, negative values of $\lambda$. In this case, the above formula tells us that $\widehat{u}_{\lambda, \kappa}$ is a linear combination of two linearly independent oscillating functions outside $[-\ell,+\ell]$. So it belongs to $L^{2}(\mathbb{R})$ (see $(22)$ ) only if both coefficients of the combination vanish, which yields

$$
\widehat{\alpha}_{\lambda, \kappa}^{ \pm}=\widehat{\beta}_{\lambda, \kappa}^{ \pm}=0 \text { for a.e. } \lambda \in \Lambda \cap(-\infty, 0) \text { and } \kappa \in K_{\lambda} \text {. }
$$

On the other hand, for positive values of $\lambda$, function $\widehat{u}_{\lambda, \kappa}$ belongs to $L^{2}(\mathbb{R})$ only if the coefficients of the unbounded exponential vanish, which yields

$$
\widehat{\alpha}_{\lambda, \kappa}^{ \pm}=0 \text { for a.e. } \lambda \in(0,+\infty) \text { and } \kappa \in K_{\lambda}=S^{1} \text {. }
$$

To sum up, we have proved that if $u$ is a finite-energy solution to (21), then its generalized Fourier transform writes as

$$
\widehat{u}_{\lambda, \kappa}(x)=\int_{\mathbb{R}} \gamma_{\lambda}\left(x-x^{\prime}\right) \widehat{f}_{\lambda, \kappa}\left(x^{\prime}\right) \mathrm{d} x^{\prime} \quad \text { for } x \in \mathbb{R} \text { and a.e. } \lambda \in \Lambda \text { and } \kappa \in K_{\lambda},
$$

and must be such that

$$
\widehat{u}_{\lambda, \kappa}(x)=0 \text { for }|x|>\ell \text { and a.e. } \lambda \in \Lambda \cap(-\infty, 0) \text { and } \kappa \in K_{\lambda} .
$$

The latter condition means that outside a section containing the support of $f$, all spectral components of $u$ associated with propagative modes must vanish. This is not surprising. Indeed, the propagative modes are those which transport energy along the waveguide axis. Hence if the corresponding spectral components did not vanish, the energy of $u$ would be infinite.

\subsection{Third step: using an analyticity argument}

Consider the expression (25) of $\widehat{u}_{\lambda, \kappa}$ for $\lambda$ in the continuous spectrum $\Lambda_{\mathrm{c}}=\left[-k_{\infty}^{2},+\infty\right)$. From (24) and the definition (17) of the generalized Fourier transform, this expression becomes

$$
\widehat{u}_{\lambda, \kappa}(x)=\int_{\mathbb{R}} \frac{\mathrm{e}^{-\sqrt{\lambda}\left|x-x^{\prime}\right|}}{2 \sqrt{\lambda}} \int_{\mathbb{R}^{2}} f\left(x^{\prime}, y\right) \overline{\Phi_{\lambda, \kappa}(y)} \mathrm{d} y \mathrm{~d} x^{\prime} \quad \text { for } x \in \mathbb{R}, \quad \lambda \in \Lambda_{\mathrm{c}}, \kappa \in S^{1} .
$$

Recall that $f$ is compactly supported. Hence the above integral is actually defined on a bounded domain, say $L \times \Omega \subset \mathbb{R} \times \mathbb{R}^{2}$. Noticing that

- for all $\kappa \in S^{1}$, the function $\left(-k_{\infty}^{2},+\infty\right) \ni \lambda \mapsto \overline{\Phi_{\lambda, \kappa}} \in L^{2}(\Omega)$ extends to a meromorphic function of $\lambda$ in the complex half plane $\operatorname{Re} \lambda>-k_{\infty}^{2}$ (see Proposition 4),

- $\lambda \mapsto \sqrt{\lambda}$ is analytic outside the branch cut (see (5)),

we infer that for all $x \in \mathbb{R}$ and $\kappa \in S^{1}$, the function $\left(-k_{\infty}^{2},+\infty\right) \ni \lambda \mapsto \widehat{u}_{\lambda, \kappa}(x) \in \mathbb{C}$ extends to a meromorphic function of $\lambda$ in the complex half plane $\operatorname{Re} \lambda>-k_{\infty}^{2}$ outside the branch cut of the complex square root. As $(26)$ tells us that if $|x|>\ell$, this function vanishes on the interval $\left(-k_{\infty}^{2}, 0\right)$, we deduce that it vanishes everywhere. To sum up, outside a section containing the support of $f$, the spectral components of $u$ associated with evanescent modes also vanish.

By the inverse generalized Fourier transform, $u$ vanishes outside a section containing the support of $f$. From the unique continuation principle [15] applied to equation (20), we conclude that $u$ vanishes outside the support of $f$. This completes the proof of Theorem 5 , and thus also of Theorem 1 .

\section{Possible extensions of Theorem 1}

In the mathematical model introduced in section 1.2, we have considered the case of a penetrable defect in a uniform waveguide. The absence of trapped modes holds for non-penetrable defects, that is, if one replaces the Helmholtz equation (1) set in the whole space by

$$
-\Delta u-k^{2} u=0 \quad \text { in } \mathbb{R}^{3} \backslash D,
$$


where $D$ denotes a compact subset of $\mathbb{R}^{3}$ which may represent the non-penetrable part of the defect. Any kind of boundary condition can be imposed on the boundary of $D$. Actually, it is not even necessary to impose a boundary condition to prove that Theorem 1 holds true in this context with the same assumptions (2)-(4) considered outside $D$. The only change in the proof lies in the first step which allows us to get rid of the defect. We simply have to choose $R>0$ such that $\bar{D} \subset B_{R}:=\left\{(x, y) \in \mathbb{R}^{3} ; x^{2}+|y|^{2}<\right.$ $\left.R^{2}\right\}$ and a function $\chi \in \mathcal{C}^{\infty}\left(\mathbb{R}^{3}\right)$ such that $\chi=0$ in $B_{R}$ and $\chi=1$ outside $B_{R+1}$. If $u \in H^{1}\left(\mathbb{R}^{3} \backslash D\right)$ is a solution to (27), then function $\chi u$ which is initially defined in $\mathbb{R}^{3} \backslash D$ can be naturally continued by 0 inside $D$ so that $\chi u \in H^{1}\left(\mathbb{R}^{3}\right)$. Moreover we have

$$
-\Delta(\chi u)-k_{\text {uni }}^{2}(\chi u)=f(u) \text { in } \mathbb{R}^{3}
$$

where

$$
f(u):=\left(k^{2}-k_{\text {uni }}^{2}\right)(\chi u)-2 \nabla \chi \cdot \nabla u-(\Delta \chi) u \in L^{2}\left(\mathbb{R}^{3}\right) .
$$

As $f(u)$ is compactly supported, Theorem 5 shows that $u$ vanishes outside the support of $f(u)$. And the fact that it vanishes also inside this support is again a consequence of the unique continuation principle. This shows that the statement of Theorem 1 remains valid for the exterior Helmholtz equation (27).

Instead of the Laplace operator, one can consider a second-order differential operator $\nabla \cdot(\alpha \nabla)$ with a variable coefficient $\alpha=\alpha(x, y)$ appearing as a localized perturbation of a function $\alpha_{\text {uni }}=\alpha_{\text {uni }}(y)$ where $\alpha$ and $\alpha_{\text {uni }}$ satisfy the same assumptions (2)-(4) as $k$ and $k_{\text {uni }}$. The absence of trapped modes then holds for the equation

$$
-\nabla \cdot(\alpha \nabla u)-k^{2} u=0 \quad \text { in } \mathbb{R}^{3},
$$

provided that the unique continuation principle applies. The proof is the same. The only change lies in the generalized Fourier transform $\mathcal{U}$ which must diagonalize the transverse part of the above operator.

A more involved extension of Theorem 1 can be obtained for the junction of two semi-infinite open waveguides. In this case, $k$ appears as a local perturbation of a function $k_{\text {abr }}$ (in the sense that $k-k_{\text {abr }}$ is compactly supported), where $k_{\mathrm{abr}}=k_{\mathrm{abr}}(x, y)$ represents the wavenumber function of an abrupt junction of two uniform waveguides described respectively by two functions $k_{\text {uni }}^{ \pm}=k_{\text {uni }}^{ \pm}(y)$ :

$$
k_{\mathrm{abr}}(x, y)=k_{\text {uni }}^{ \pm}(y) \quad \text { if } x \in \mathbb{R}^{ \pm} .
$$

In this context, the general strategy of the proof of Theorem 1 still applies but its implementation becomes far more intricate, notably because of the use of two different generalized Fourier transforms on both sides of the junction. One can follow the same steps as in [6] (which deals with the case of a two-dimensional junction of step-index waveguides).

On the other hand, the question of the existence of trapped modes in open curved waveguides seems to be open, as well as the case of locally perturbed periodic waveguides. Works aiming to apply the ideas explained in the present paper to these situations are in progress.

\section{Funding}

POEMS lab (Propagation d'Ondes: Etude Mathématique et Simulation) is a mixed research team (UMR 7231) funded by CNRS (Centre National de la Recherche Scientifique), ENSTA ParisTech (Ecole Nationale Supérieure de Techniques Avancées) and INRIA (Institut National de Recherche en Informatique et en Automatique).

\section{References}

[1] Aubin, J.-P. (2000) Applied Functional Analysis. John Wiley \& Sons.

[2] Berezansky, Y.M., Sheftel, Z.G. \& Us, G.F. (1996) Functional Analysis. Vol. 2. Birkhäuser.

[3] Bonnet-Ben Dhia, A.-S., Dakhia, G., Hazard, C. \& Chorfi, L. (2009) Diffraction by a defect in an open waveguide : a mathematical analysis based on a modal radiation condition, SIAM J. Appl. Math., 70, 677-693. 
[4] Bonnet-Ben DhiA, A.-S \& Djellouli, R. (1994) High-frequency asymptotics of guided modes in optical fibers, IMA J. Appl. Math., 52, 271-287.

[5] Bonnet-Ben DhiA, A.-S., Duterte, J. \& Joly, P. (1999) Mathematical analysis of elastic surface waves in topographic waveguides, Mathematical Models and Methods in Applied Sciences, 9, $755-798$.

[6] Bonnet-Ben Dhia, A.-S., Goursaud, B. \& Hazard, C. (2011) Mathematical analysis of the junction of two acoustic open waveguides, SIAM J. Appl. Math., 71, 2048-2071.

[7] Bonnet-Ben Dhia, A.-S. \& Joly, P. (2001) Mathematical analysis and numerical approximation of optical waveguides. In: G. BAO, L. Cowsar And W. MAsters, eds. Mathematical Modeling in Optical Science, SIAM, 273-324.

[8] Colton, D. \& Kress, R. (1992) Inverse Acoustic and Electromagnetic Scattering Theory. SpringerVerlag.

[9] Dauge, M. \& Raymond, N. (2012) Plane waveguides with corners in the small angle limit, $J$. Math. Phys., 53, 123529.

[10] DeBiÈvre, S. \& PravicA, D.W. (1992) Spectral analysis for optical fibres and stratified fluids II: Absence of eigenvalues, Communications in Partial Differential Equations, 17, 69-97.

[11] Duclos, P. \& Exner, P. (1995) Curvature-induced bound states in quantum waveguides in two and three dimensions, Rev. Math. Phys., 7, 73-102.

[12] Gralak, B. \& Maystre, D. (2012) Negative index materials and time-harmonic electromagnetic field, Comptes Rendus Physique, 13, 786-799.

[13] Hazard, C. \& Loret, F. (2007) Generalized eigenfunction expansions for conservative scattering problems with an application to water waves, Proc. Roy. Soc. Edinburgh Sect. A, 137, 995-1035.

[14] HAzARD, C. (2009) A distribution framework for the generalized Fourier transform associated with a Sturm-Liouville operator, Research report of INRIA, 6885, available online from http: //hal.inria.fr/inria-00371350/fr/.

[15] Le Rousseau, J. \& Lebeau, G. (2011) On Carleman estimates for elliptic and parabolic operators. Applications to unique continuation and control of parabolic equations, ESAIM: Control, Optimisation and Calculus of Variations, doi:10.1051/cocv/2011168.

[16] Linton, C.M. \& McIver, P. (2007) Embedded trapped modes in water waves and acoustics, Wave Motion, 45, 16-29.

[17] Littman, W. (1986) Spectral properties of the Laplacian in the complement of a deformed cylinder, Arch. Ration. Mech. Anal., 96, 319-325.

[18] Shenk, N. \& Thoe, D. (1971) Eigenfunction expansions and scattering theory for perturbations of $-\Delta$, J. Math. Anal. Appl., 36, 313-351.

[19] Steinberg, S. (1968) Meromorphic families of compact operators, Arch. Rational Mech. Anal., 31, $372-380$.

[20] Vassalo, C. (1991) Optical Waveguide Concepts. Elsevier.

[21] Weder, R. (1988) Absence of eigenvalues of the acoustic propagator in deformed wave guides, Rocky Mountain J. Math., 18, 495-503.

[22] Weder, R. (1991) Spectral and Scattering Theory for Wave Propagation in Perturbed Stratified Media. Appl. Math. Sci., 87. Springer-Verlag. 